\title{
Optimal Row-Column Design for Three Treatments
}

\author{
J. P. Morgan and Valentin Parvu \\ Department of Statistics, Virginia Tech
}

$22^{\text {nd }}$ February 2006

Abstract: The A-optimality problem is solved for three treatments in a row-column layout. Depending on the numbers of rows and columns, the requirements for optimality can be decidedly counterintuitive: replication numbers need not be as equal as possible, and trace of the information matrix need not be maximal. General rules for comparing $3 \times 3$ information matrices for their A-behavior are also developed, and the A-optimality problem is also solved for three treatments in simple block designs.

Key words and phrases: A-optimality, row-column blocking, uniform treatment assignment.

\section{Introduction}

The classical row-column design problem begins with $p q$ experimental units for use in comparing some $v$ treatments of interest. Visualized as a $p \times q$ array, these units are subject to two identifiable, systematic sources of heterogeneity, represented by the rows and the columns of the array. Treatments will be assigned to the units, with the goal of evaluating treatment differences as efficiently as possible after removing row and column variation.

Latin square, Youden, generalized Youden and pseudo-Youden designs are examples of optimal rowcolumn designs (Kiefer, 1975; Cheng, 1981). These designs are all subject to strict combinatorial requirements, with the consequence that in the universe of all possible row-column experiments $(v, p, q)$, they comprise a very small fraction. Optimality progress outside of these combinatorially 
rarefied settings has been at best sporadic, with most known results being for the E-criterion; see Jacroux (1985, 1986, 1987, 1990), Bagchi and van Berkum (1991), Singh and Gupta (1991), Das (1993), Bagchi (1996), and Parvu and Morgan (2005). It has long been known that optimal, equireplicate block designs ( $v$ treatments in $b$ blocks of size $k$ ) can be arranged into optimal rowcolumn designs (of size $b \times k$ ) whenever $b$ is a multiple of $v$; the underlying technical result was formalized by Magda (1980). Bagchi and Shah (1989) managed to find strong optimality results, including A-optimality, for a class of designs in which each of the rows and the columns are linked block designs. Sonnemann (1985) solved the row-column optimality problem for $v=2$.

To the authors' knowledge, Sonnemann (1985) is the only published work to have established Aoptimality in a row-column setting when $p q$ is not a multiple of $v$, that is, when equal replication is not possible. Indeed, knowledge of A-optimal row-column designs, aside from the Youden families and those directly derivable from block designs as described above, is quite thin. This is general evidence that these are very difficult problems. Specific evidence can be found in a reading of Kiefer's (1975) intricate work on A-optimality for the GYDs, which with their considerable symmetry would otherwise appear to be excellent candidates for a simpler, relatively transparent proof. One reason for the difficulties, and as noted by Das and Dey (1992), is that GYDs need not have maximal trace of the information matrix. This is contrary to intuition from the much better understood problem of one-way blocking, and is a major reason why the elegant (though also sometimes difficult) arguments there have not translated well. More generally for row-column settings, binarity in rows and columns need not produce maximal trace of the information matrix, and maximal trace need not be a property of optimal designs.

This paper tackles, and solves, the A-optimality problem for $v=3$ treatments in a $p \times q$ row-column experiment. When $p$ and $q$ are such that equal replication is not possible, A-optimal designs sometimes do, and sometimes do not, have replications as close to equal as possible. Section 2 provides relevant optimality background, then develops A-bounds for the general (not limited to row-column) problem for three treatments. These bounds help solve the simpler block design problem in section 3, then are used in section 4 as the backbone on which fairly intricate optimality calculations comprising the main proof are built. Discussion follows in section 5 . With no loss of generality, $p \leq q$ is assumed throughout. 


\section{Terminology and preliminary results}

A row-column design has two component block designs, consisting of the $p$ row blocks of size $q$, and the $q$ column blocks of size $p$. Because each of these components comes into play, this discussion begins with block design terminology. For any block design, let $n_{i j}$ denote the number of experimental units to which treatment $i$ is assigned in block $j$. Block (and row-column, and other) designs are evaluated in terms of the $v \times v$ treatment information matrix, usually denoted by $C_{d}$ for design $d$ and also called the $C$-matrix. For a block design with $v$ treatments in $b$ blocks of size $k$, the $C$-matrix is

$$
C_{d}=\operatorname{diag}\left(r_{1}, \ldots, r_{v}\right)-\frac{1}{k} N N^{\prime}
$$

where $N=\left(n_{i j}\right)$ is the matrix of block-wise assignment numbers and $r_{i}$ is the total replication for treatment $i$. While the $r_{i}$ and $n_{i j}$ (and other quantities to appear) are certainly functions of the design $d$, this fact will not be reflected in the notation unless needed for clarity.

Definition 2.1. The assignment of treatment $i$ to blocks of size $k$ is binary if $n_{i j} \in\{0,1\}$ for all $j$. The assignment of treatment $i$ is generalized binary if $\left|n_{i j}-k / v\right|<1$ for all $j$.

As a verbal shorthand, "treatment $i$ is binary" will be used for "the assignment of treatment $i$ is binary." A block design is said to be binary (generalized binary) if all the treatments are binary (generalized binary). Generalized binary designs maximize the trace of the $C$-matrix in the class of designs for one blocking factor with fixed $(v, b, k)$.

Definition 2.2. In a block design, the assignment of treatment $i$ is said to be uniform if $\left|n_{i j}-n_{i j^{\prime}}\right| \leq$ 1 for any blocks $j$ and $j^{\prime}$.

As before, "treatment $i$ is uniform" is shorthand for "the assignment of treatment $i$ is uniform." A design is said to be uniform if all the treatments are uniform. If treatment $i$ is generalized binary, it is uniform. Also, if treatment $i$ is uniform with a replication number $r_{i}$ such that $b\left[\operatorname{int}\left(\frac{k}{v}\right)\right] \leq r_{i} \leq b\left[\operatorname{int}\left(\frac{k}{v}\right)+1\right]$ and $v \nmid k$, treatment $i$ is also generalized binary.

Turning now to row-column designs, let $N_{1}=\left(n_{i j 1}\right)\left(N_{2}=\left(n_{i j 2}\right)\right)$ be the $v \times p(v \times q)$ incidence matrix between the $v$ treatments and the $p$ row blocks ( $q$ column blocks). The information matrix 
for row-column design $d$ is

$$
C_{d}=\operatorname{diag}\left(r_{1}, \ldots, r_{v}\right)-\frac{1}{q} N_{1} N_{1}^{\prime}-\frac{1}{p} N_{2} N_{2}^{\prime}+\frac{1}{p q} r r^{\prime}
$$

where $r$ is the $v \times 1$ replication vector. Imbedded in (2) is the column component information matrix $\operatorname{diag}\left(r_{1}, \ldots, r_{v}\right)-\frac{1}{p} N_{2} N_{2}^{\prime}$. Indeed, $C_{d}$ of $(2)$ can be written $C_{d}=\operatorname{diag}\left(r_{1}, \ldots, r_{v}\right)-\frac{1}{p} N_{2} N_{2}^{\prime}-X$ where $X=\frac{1}{q} N_{1} N_{1}^{\prime}-\frac{1}{p q} r r^{\prime}$ is non-negative definite. It follows that for any design $d, \Phi\left(\operatorname{diag}\left(r_{1}, \ldots, r_{v}\right)-\right.$ $\left.\frac{1}{p} N_{d} N_{d}^{\prime}\right) \leq \Phi\left(C_{d}\right)$ for any optimality criterion $\Phi$ as defined below, with equality precisely when $X$ is null. Nullity of $X$ occurs when the row assignment numbers $n_{i j 1}$ are constant in the row index $j$, which can be incompatible with replicating the treatments as equally as possible, and so also with generalized binarity in rows and in columns.

The $i$ th diagonal element of $C_{d}$ for a row-column design is

$$
c_{i}=r_{i}-\frac{1}{q} \sum_{j=1}^{p} n_{i j 1}^{2}-\frac{1}{p} \sum_{j=1}^{q} n_{i j 2}^{2}+\frac{1}{p q} r_{i}^{2} .
$$

If $\sum_{j=1}^{p} n_{i j 1}=r_{i}$ is fixed, then $\sum_{j=1}^{p} n_{i j 1}^{2}$ is minimized when the $n_{i j 1}$ 's are as close to each other as possible (i.e. treatment $i$ is uniform in rows); a similar statement applies to columns. Thus, given that treatment $i$ has replication $r_{i}$, the diagonal element $c_{i}$ will be maximized if treatment $i$ is uniform in rows and in columns (see Definition 2.2). The trace of $C_{d}$ is maximized for given replications if each treatment is uniform in rows and in columns, in which case each treatment as well as $d$ itself is said to be uniform.

Define the function $h$ of positive integers $x$ and $y$ by

$$
h(x, y)=x+(2 x-y) \operatorname{int}\left(\frac{x}{y}\right)-y\left[\operatorname{int}\left(\frac{x}{y}\right)\right]^{2} .
$$

The value $h(x, y)$ is the minimum of $\sum_{j=1}^{y} a_{j}^{2}$ subject to $\sum_{j=1}^{y} a_{j}=x$ and $a_{j}$ 's nonnegative integers. For any design where treatment $i$ is uniform in rows and columns,

$$
c_{i}=r_{i}-\frac{1}{q} h\left(r_{i}, p\right)-\frac{1}{p} h\left(r_{i}, q\right)+\frac{1}{p q} r_{i}^{2}
$$

denoted $c_{i}\left(r_{i}\right)$ when thought of as a function of $r_{i}$. A related useful quantity is $\Delta h$, the difference between $h(x+1, y)$ and $h(x, y)$. Simple manipulation (see Morgan, 1997) gives

$$
\Delta h(x, y)=h(x+1, y)-h(x, y)=1+\frac{2}{y}\left(x-x_{(y)}\right)
$$


where $x_{(y)}=x(\bmod y)$. If $x$ is allowed to be any positive real number, $h(x, y)$ is a continuous, increasing function of $x$, differentiable at all points except $x=s y$ for integers $s$. Thus $c_{i}\left(r_{i}\right)$ in $(5)$ is also a continuous function of $r_{i}$, differentiable at all points except $r_{i}=s p$ or $s q$, for integers $s$.

Lemma 2.1. If $r_{i} \leq \frac{(p-1)(q-1)}{2}$, the function $c_{i}\left(r_{i}\right)$ is an increasing function of $r_{i}$.

Proof. Using (5) and (6), one gets:

$$
\begin{aligned}
p q\left[c_{i}\left(r_{i}+1\right)-c_{i}\left(r_{i}\right)\right] & =p q-p \Delta h\left(r_{i}, p\right)-q \Delta h\left(r_{i}, q\right)+\left(2 r_{i}+1\right) \\
& =p q+1-2 r_{i}-(p+q)+2\left[\left(r_{i}\right)_{(p)}+\left(r_{i}\right)_{(q)}\right] \\
& \geq(p-1)(q-1)-2 r_{i}
\end{aligned}
$$

For block designs, $c_{i}\left(r_{i}\right)=r_{i}-\frac{1}{k} h\left(r_{i}, b\right)$ for uniform treatment $i$, which is increasing in $r_{i} \leq \frac{b(k-1)}{2}$. Most commonly used optimality criteria are functions of the $v-1$ positive eigenvalues $z_{1} \geq z_{2} \geq$ $\cdots \geq z_{v-1}\left(>z_{v}=0\right)$ of the $C$-matrix. A typical criterion $\Phi$ is

$$
\Phi\left(C_{d}\right)=\sum_{i=1}^{v-1} f\left(z_{i}\right)
$$

for some convex, non-increasing $f$. A $\Phi$-optimal design minimizes $\Phi\left(C_{d}\right)$ with respect to $d$. Of chief concern here is the A-criterion, for which $f(x)=\frac{1}{x}$. Minimization of $\Phi_{A}$ is equivalent to minimizing the average variance of estimators of any $v-1$ orthogonal contrasts.

A useful technique for establishing optimality bounds was introduced by Constantine (1981). Given $C_{d}$, define $\bar{C}_{d}$ by

$$
\bar{C}_{d}=\frac{1}{s} \sum_{i=1}^{s} P_{i}^{\prime} C_{d} P_{i},
$$

where the $P_{i}$ are a collection of $s \leq v$ ! permutation matrices. If the nonzero eigenvalues of $\bar{C}_{d}$ are $\bar{z}_{1} \geq \bar{z}_{2} \geq \cdots \geq \bar{z}_{v-1}$, then $\sum_{i=1}^{v-1} f\left(z_{i}\right) \geq \sum_{i=1}^{v-1} f\left(\bar{z}_{i}\right)$ for every convex $f$ (see 9.G.1 in Marshall and Olkin, 1979).

The remainder of this section will specialize to $v=3$. Since the $3 \times 3$ information matrix is symmetric with row and column sums of zero, it can be written entirely in terms of its diagonal 
elements:

$$
C_{d}=\left(\begin{array}{ccc}
c_{1} & \frac{1}{2}\left(-c_{1}-c_{2}+c_{3}\right) & \frac{1}{2}\left(-c_{1}+c_{2}-c_{3}\right) \\
\frac{1}{2}\left(-c_{1}-c_{2}+c_{3}\right) & c_{2} & \frac{1}{2}\left(c_{1}-c_{2}-c_{3}\right) \\
\frac{1}{2}\left(-c_{1}+c_{2}-c_{3}\right) & \frac{1}{2}\left(c_{1}-c_{2}-c_{3}\right) & c_{3}
\end{array}\right) .
$$

The positive eigenvalues of (8) are

$$
z_{1}=\frac{1}{2}\left[\sum c_{i}+\sqrt{2 \sum_{i<j}\left(c_{i}-c_{j}\right)^{2}}\right] \text { and } z_{2}=\frac{1}{2}\left[\sum c_{i}-\sqrt{2 \sum_{i<j}\left(c_{i}-c_{j}\right)^{2}}\right]
$$

An $A$-optimal design minimizes

$$
\Phi_{A}\left(C_{d}\right)=\frac{1}{z_{1}}+\frac{1}{z_{2}}=\frac{4}{3}\left(c_{1}+c_{2}+c_{3}-2 \frac{c_{1}^{2}+c_{2}^{2}+c_{3}^{2}}{c_{1}+c_{2}+c_{3}}\right)^{-1}
$$

which is equivalent to maximizing the quantity $A$ given by

$$
A=c_{1}+c_{2}+c_{3}-2 \frac{c_{1}^{2}+c_{2}^{2}+c_{3}^{2}}{c_{1}+c_{2}+c_{3}} .
$$

The expression in (10) looks inviting, but is far from simple to manipulate. Several lemmas, needed in the optimality proofs of sections 3 and 4 , are developed next. Let $d(x, y, z)$ denote a design whose $C$-matrix has diagonal elements $c_{1}=x, c_{2}=y$, and $c_{3}=z$. Since any $C$-matrix is nonnegative definite, $x, y$, and $z$ are non-negative numbers.

Lemma 2.2. Given a design $d^{*}\left(x^{*}, y^{*}, z^{*}\right)$, consider a competitor $d(x, y, z)$. If

(i) $x^{*}+y^{*}+z^{*} \geq x+y+z$, and

(ii) $x^{* 2}+y^{* 2}+z^{* 2}<x^{2}+y^{2}+z^{2}$

then $d^{*}$ is A-superior to $d$.

Lemma 2.2 follows directly from (10).

Lemma 2.3. If $x \geq y \geq z$, then $A$ increases as $x$ increases if and only if $x<-(y+z)+$ $2 \sqrt{y^{2}+y z+z^{2}}$. A sufficient condition for this is $x<(\sqrt{3}-1)(y+z)$. Also, $A$ is an increasing function of $y$ and $z$ for $x \geq y \geq z$.

Proof. The partial derivative of $A$ with respect to $x$ is:

$$
\frac{\partial A}{\partial x}=1-2 \frac{2 x(x+y+z)-x^{2}-y^{2}-z^{2}}{(x+y+z)^{2}}=\frac{4 y^{2}+4 z^{2}+4 y z-(x+y+z)^{2}}{(x+y+z)^{2}}
$$


which is positive if and only if the numerator is positive, that is, if and only if $4 y^{2}+4 z^{2}+4 y z>(x+y+z)^{2} \Leftrightarrow x+y+z<2 \sqrt{y^{2}+y z+z^{2}} \Leftrightarrow x<-(y+z)+2 \sqrt{y^{2}+y z+z^{2}}$.

Since $y^{2}+y z+z^{2} \geq \frac{3}{4}(y+z)^{2}$, with equality when $y=z$, the sufficient condition is established.

Likewise the partial derivative of $A$ with respect to $y$ is

$$
\frac{\partial A}{\partial y}=\frac{4 x^{2}+4 z^{2}+4 x z-(x+y+z)^{2}}{(x+y+z)^{2}}=\frac{\left(x^{2}-y^{2}\right)+2 x(x-y)+2 z(x-y)+3 z^{2}}{(x+y+z)^{2}} .
$$

which is obviously positive because $x \geq y$.

Lemma 2.4. Given a uniform design $d^{*}\left(x^{*} \geq y^{*} \geq z^{*}\right)$, consider a nonuniform design $d(x, y, z)$ with the same treatment replications as $d^{*}$. If $x^{*}<(\sqrt{3}-1)\left(y^{*}+z^{*}\right)$, then $d$ is A-inferior to $d^{*}$.

Proof. Since for fixed replications uniform designs maximize the diagonal elements of the $C$-matrix, $x^{*} \geq x, y^{*} \geq y$, and $z^{*} \geq z$, with at least one strict inequality. Now apply lemma 2.3 .

Lemma 2.5. Given a design $d^{*}\left(x^{*}, y^{*}, y^{*}\right)$ with $x^{*}>y^{*}$, consider a competitor $d(x, y, y)$. If

(i) $x^{*}<2(\sqrt{3}-1) y^{*}$,

(ii) $x+2 y \leq x^{*}+2 y^{*}$, and

(iii) $y<y^{*}$

then $d$ is A-inferior to $d^{*}$.

Proof. If $x \leq y^{*}$, then $d(x, y, y)$ is $A$-inferior to $d\left(y^{*}, y^{*}, y^{*}\right)$ since the latter C-matrix is completely symmetric of higher trace, and by $(i)$ and lemma $2.3, d\left(y^{*}, y^{*}, y^{*}\right)$ is $A$-inferior to $d^{*}$.

If $y^{*}<x \leq x^{*}$, (iii) and lemma $2.3 \Rightarrow d(x, y, y)$ is $A$-inferior to $d\left(x, y^{*}, y^{*}\right)$, which is inferior to $d^{*}$.

If $x>x^{*}$, write $x=x^{*}+a$, and $y=y^{*}-\frac{b}{2}$. From $(i i)$ and (iii) it follows that $b \geq a>0$. Let $A^{*}$ and $A$ be computed as in (10) for designs $d^{*}\left(x^{*}, y^{*}, y^{*}\right)$ and $d(x, y, y)$. Then

$$
\begin{aligned}
A^{*}-A & =\left(x^{*}+2 y^{*}\right)-\frac{2\left(x^{* 2}+2 y^{* 2}\right)}{x^{*}+2 y^{*}}-\left(x^{*}+2 y^{*}+a-b\right)+\frac{2\left[\left(x^{*}+a\right)^{2}+2\left(y^{*}-b / 2\right)^{2}\right]}{x^{*}+2 y^{*}+a-b} \\
& =b-a-\frac{2\left(x^{* 2}+2 y^{* 2}\right)}{x^{*}+2 y^{*}}+\frac{2\left(x^{* 2}+2 y^{* 2}+2 x^{*} a-2 y^{*} b+a^{2}+b^{2} / 2\right)}{x^{*}+2 y^{*}+a-b} \\
& =\frac{x^{* 2}(a+3 b)-8 y^{* 2} a+4 x^{*} y^{*} a+x^{*}\left(a^{2}+2 a b\right)+y^{*}\left(2 a^{2}+4 a b\right)}{\left(x^{*}+2 y^{*}\right)\left(x^{*}+2 y^{*}+a-b\right)}
\end{aligned}
$$


which has positive denominator. Since $b \geq a>0$ and $x^{*}>y^{*}$, then $x^{* 2}(a+3 b)>4 a y^{* 2}$ and $4 x^{*} y^{*} a>4 a y^{* 2}$, and thus $\left(x^{*}+2 y^{*}\right)\left(x^{*}+2 y^{*}+a-b\right)\left(A^{*}-A\right)>x^{*}\left(a^{2}+2 a b\right)+y^{*}\left(2 a^{2}+4 a b>0\right.$

Lemma 2.6. Given a design $d^{*}\left(x^{*}, x^{*}, y^{*}\right)$ with $x^{*}>y^{*}$, consider a competitor $d(x, x, y)$. If

(i) $2 x+y \leq 2 x^{*}+y^{*}$, and

(ii) $y<y^{*}$

then $d$ is A-inferior to $d^{*}$.

Proof. First notice that $x<-(x+y)+2 \sqrt{x^{2}+x y+y^{2}}$ for any $x$ and $y$, so for $x \leq x^{*}$, lemma 2.3 implies $d(x, x, y)$ is $A$-inferior to $d\left(x^{*}, x^{*}, y\right)$, which by $(i i)$ and lemma 2.3 is $A$-inferior to $d^{*}$.

If $x>x^{*}$, write $x=x^{*}+\frac{a}{2}$, and $y=y^{*}-b$. Similarly to the proof of lemma 2.5,

$$
\begin{aligned}
A^{*}-A & =b-a-\frac{2\left(2 x^{* 2}+y^{* 2}\right)}{2 x^{*}+y^{*}}+\frac{2\left(2 x^{* 2}+y^{* 2}+2 x^{*} a-2 y^{*} b+b^{2}+a^{2} / 2\right)}{2 x^{*}+y^{*}+a-b} \\
& =\frac{8 b x^{* 2}-(3 a+b) y^{* 2}-4 b x^{*} y^{*}+x^{*}\left(2 b^{2}+4 a b\right)+y^{*}\left(b^{2}+2 a b\right)}{\left(2 x^{*}+y^{*}\right)\left(2 x^{*}+y^{*}+a-b\right)}
\end{aligned}
$$

Since $x^{*}>y^{*}$, and by $(i)$ and $(i i), b \geq a>0$, it follows that $(3 a+b) y^{* 2}<4 b x^{* 2}$ and $4 b x^{*} y^{*}<4 b x^{* 2}$ and thus $\left(2 x^{*}+y^{*}\right)\left(2 x^{*}+y^{*}+a-b\right)\left(A^{*}-A\right)>x^{*}\left(2 b^{2}+4 a b\right)+y^{*}\left(b^{2}+2 a b\right)>0$

\section{A-optimal Designs with One Blocking Factor}

As a first application of results in section 2, this section will determine A-optimality for three treatments in simple block designs. Here and in section 4 it is assumed that $r_{1} \geq r_{2} \geq r_{3}$. The diagonal elements of the $C$-matrix (1) can be written as:

$$
c_{i}=r_{i}-\frac{1}{k} \sum_{j=1}^{b} n_{i j}^{2},
$$

which if treatment $i$ is uniform is

$$
c_{i}=r_{i}-\frac{1}{k} h\left(r_{i}, b\right)
$$

where $h$ is defined in (4).

Theorem 3.1. A block design for three treatments in b blocks of size $k$ is A-optimal if and only if it is generalized binary with $r_{3} \geq r_{1}-1$. 
Proof. For $b k \equiv 0(\bmod 3)$ the proposed design is just a BBD as defined by Kiefer (1975), so is universally optimal. Uniqueness follows from two considerations. Any competitor $d$ with the same trace, but for which $C_{d}$ is not completely symmetric, is eliminated by lemma 2.2. Symmetrizing over all treatments (via (7)) any competitor $d$ with smaller trace produces another, obviously inferior, information matrix.

For $b k \equiv 2(\bmod 3)$, write $\bar{r}=\frac{b k-2}{3}$ and denote the proposed generalized binary design with replications $\left(r_{1}, r_{2}, r_{3}\right)=(\bar{r}+1, \bar{r}+1, \bar{r})$ by $d^{0}=d^{0}\left(c_{1}^{0}, c_{2}^{0}, c_{3}^{0}\right)$. Given a competitor design $d\left(c_{1}, c_{2}, c_{3}\right)$ with $r_{3}<\bar{r}$, symmetrize on treatments 1 and 2 as in (7) to produce $\bar{d}\left(\bar{c}_{1}, \bar{c}_{1}, c_{3}\right)$, where $\bar{c}_{1}=\frac{c_{1}+c_{2}}{2}$. Since $\operatorname{tr}\left(C_{d}\right)=\operatorname{tr}\left(C_{\bar{d}}\right)$ and $c_{1}^{2}+c_{2}^{2} \geq 2 \bar{c}_{1}^{2}$, it follows from lemma 2.2 that $A_{d} \leq A_{\bar{d}}$. Treatment 3 is generalized binary and has higher replication in $d^{0}$ than in $d$, so $c_{3}^{0}>c_{3}$. Also, $C_{d^{0}}$ has maximum trace, so the conditions of lemma 2.6 are satisfied. Therefore $d^{0}$ is $A$-superior to $\bar{d}$, which is $A$-superior (or equal) to $d$.

Since designs with $r_{3}<\bar{r}$ are $A$-inferior to $d^{0}$, the remaining candidates have replications $(\bar{r}+2, \bar{r}, \bar{r})$ or $(\bar{r}+1, \bar{r}+1, \bar{r})$. For a generalized binary design with these replications, $c_{1}$ is maximum and $c_{2}+c_{3}$ is minimum for $(\bar{r}+2, \bar{r}, \bar{r})$; call this design $d^{\prime}$. Using (12) and (4), $c_{1}$ and $c_{2}=c_{3}$ can be calculated for $d^{\prime}$ as explicit functions of $b$ and $k$, from which $c_{1}<2(\sqrt{3}-1) c_{2}$ easily follows. Lemma 2.4 now eliminates all nonbinary competitors, leaving only $d^{0}$ and $d^{\prime}$, which have equal trace. Equation (10) says the better of these two has smaller $\sum_{i} c_{i}^{2}$, easily seen to be $d^{0}$.

The proof for $b k \equiv 1(\bmod 3)$ follows similar steps, so is not given here.

Theorem 3.1 offers no surprises. A-optimal block designs have (M,S)-property, that is, they maximize trace, and among maximum trace designs they minimize $\operatorname{tr}\left(C_{d}^{2}\right)$ (compare conjecture 3, Shah and Sinha, 1989, page 60). The surprise, which underscores the non-triviality of Theorem 3.1, is that these designs need not be optimal in other useful senses; see Parvu and Morgan (2005). Cheng (1979a) solved the optimality problem for $v=4$ treatments in incomplete $(k<4)$ blocks.

\section{A-optimal Row-Column Designs}

The main result of this paper is now stated. 
Theorem 4.1. The A-optimal row-column designs for three treatments are uniform, with replications as specified in Table 1.

Table 1: Replications for $A$-optimal row-column designs $(p \leq q)$

\begin{tabular}{|c|c|c|c|c|c|}
\hline$p(\bmod 3)$ & $q(\bmod 3)$ & range for $q$ & $r_{1}$ & $r_{2}$ & $r_{3}$ \\
\hline 0 & $0,1,2$ & - & $\frac{p q}{3}$ & $\frac{p q}{3}$ & $\frac{p q}{3}$ \\
\hline $0,1,2$ & 0 & - & $\frac{p q}{3}$ & $\frac{p q}{3}$ & $\frac{p q}{3}$ \\
\hline 1 & 1 & - & $\frac{p(q+2)}{3}$ & $\frac{p(q-1)}{3}$ & $\frac{p(q-1)}{3}$ \\
\hline \multirow{3}{*}{1} & \multirow{3}{*}{2} & $q \geq 2 p$ & $\frac{p(q+1)}{3}$ & $\frac{p(q+1)}{3}$ & $\frac{p(q-2)}{3}$ \\
\hline & & $q<2 p, q$ even & $\frac{q(2 p+1)}{6}$ & $\frac{q(2 p+1)}{6}$ & $\frac{q(p-1)}{3}$ \\
\hline & & $q<2 p, q$ odd & $\frac{q(2 p+1)+3}{6}$ & $\frac{q(2 p+1)-3}{6}$ & $\frac{q(p-1)}{3}$ \\
\hline \multirow{3}{*}{2} & \multirow{3}{*}{1} & $\begin{array}{c}q \geq \frac{3 p+2}{2}, p \text { even, or } \\
\quad q \geq \frac{3 p-1}{2}, p \text { odd }\end{array}$ & $\frac{p q+1}{3}$ & $\frac{p q+1}{3}$ & $\frac{p q-2}{3}$ \\
\hline & & $q<\frac{3 p+2}{2}, p$ even & $\frac{p(2 q+1)}{6}$ & $\frac{p(2 q+1)}{6}$ & $\frac{p(q-1)}{3}$ \\
\hline & & $q<\frac{3 p-1}{2}, p$ odd & $\frac{p(2 q+1)+3}{6}$ & $\frac{p(2 q+1)-3}{6}$ & $\frac{p(q-1)}{3}$ \\
\hline 2 & 2 & - & $\frac{p q+2}{3}$ & $\frac{p q-1}{3}$ & $\frac{p q-1}{3}$ \\
\hline
\end{tabular}

A complete proof of Theorem 4.1 covers in excess of 50 pages in the second author's dissertation (Parvu, 2004). This length is due in large measure to the idiosyncratic behavior of the quantity $A$ defined in (10). If the number of experimental units is a multiple of 3 , or if $p$ and $q$ are both 2 $(\bmod 3)$, then the three replication numbers are as close as possible. But as can be seen in Table 1, replications for A-optimality otherwise depend non-trivially on the $(\bmod 3)$ values of $p$ and $q$ as well as the absolute difference $|p-q|$. Trace of the information matrix also varies, achieving the largest possible value only in the first four lines (of ten) in the table. Maximizing $A$ is a correspondingly intricate task. Bounding arguments are useful, but only up to a point, after which $A$ must be worked with directly.

When either $p$ or $q$ is a multiple of 3 , the A-optimal (indeed, universally optimal) designs are generalized Youden designs. This result has previously been given by Kiefer (1975), and Cheng (1979b) has established existence. These are the only equireplicate cases. 
A detailed proof for $(p, q) \equiv(1,2)(\bmod 3)$ will be given here. Proofs for the other cases where equal replication is not possible can be found in Parvu (2004). They use the same machinery and the same general breakdown into cases as shown here, so that the technical problems encountered are fairly represented by this proof.

When equireplicated designs do not exist, the general strategy is:

(i) eliminate replication numbers outside of some neighborhood of $\bar{r}=\operatorname{int}\left[\frac{p q}{3}\right]$ by comparison to a selected design (which may or may not be A-optimal)

(ii) for replications in the neighborhood determined in $(i)$, eliminate nonuniform designs relative to uniform designs

(iii) now examine the behavior of $A$ in that neighborhood, which given uniformity is solely a function of $\left(r_{1}, r_{2}, r_{3}\right)$.

Proof. The steps of this proof are best followed with an algebraic manipulator, such as Maple or Mathematica, in hand. Remember $p \leq q$ always.

For $(p, q) \equiv(1,2)(\bmod 3)$ let $d^{*}$ be a uniform design with replications as given in Table 1 . The task of showing A-optimality of $d^{*}$ will be divided into two parts, depending on the ratio $\frac{q}{p}$. Each part will be subdivided into several steps as in the general strategy outlined in (13). More of the detail will be shown in Part I than in Part II.

Part I: $\mathbf{q} \geq \mathbf{2 p}$. The smallest possible $(p, q)$ are $(4,8)$, and regardless of $(p, q), d^{*}$ is of maximal trace (since rows are permutations of one another, and the design is generalized binary in columns). Let any competitor design $d$ have replications $r_{1} \geq r_{2} \geq r_{3}$. The steps of the general strategy (13) are implemented as

1. Eliminate all designs with $r_{3}<\frac{p(q-2)}{3}$ and so with $r_{1}>\frac{p(q+4)}{3}$.

2. Eliminate all nonuniform designs with $r_{i} \in\left[\frac{p(q-2)}{3}, \frac{p(q+4)}{3}\right]$ for all $i$.

3. Eliminate all remaining designs with $r_{1}>\frac{p(q+1)}{3}$.

4. Show that among uniform designs with replications $r_{i} \in\left[\frac{p(q-2)}{3}, \frac{p(q+1)}{3}\right], d^{*}$ is $A$-optimal.

Step 1. Consider a design $d$ with $r_{3}<\frac{p(q-2)}{3}$, and $c_{1}, c_{2}, c_{3}$ the diagonal elements of $C_{d}$. Create the symmetrized design $\bar{d}$ with $\bar{c}_{3}=c_{3}$ and $\bar{c}_{1}=\bar{c}_{2}=\frac{c_{1}+c_{2}}{2}$. Design $\bar{d}$ is $A$-inferior to $d^{*}$ by lemma 
2.6, since $c_{3}<c_{3}^{*}$ by lemma 2.1 , and $d^{*}$ is of maximal trace. Using (7), design $d$ is $A$-inferior to $\bar{d}$. All remaining competitors must have $r_{i} \in\left[\frac{p(q-2)}{3}, \frac{p(q+4)}{3}\right]$. Note this step does not require $q \geq 2 p$.

Step 2. The second step eliminates nonuniform designs with replications in the neighborhood just determined. Use of lemma 2.4 requires showing that $(\sqrt{3}-1)\left(c_{2}+c_{3}\right)-c_{1} \geq 0$, where the $c_{i}$ 's are the diagonal elements of a uniform design with all $r_{i} \in\left[\frac{p(q-2)}{3}, \frac{p(q+4)}{3}\right]$. Write $r_{1}=\frac{p(q+4)}{3}-x$ for some $0 \leq x \leq \frac{4 p-1}{3}, r_{3}=\frac{p(q-2)}{3}+u$ for some $0 \leq u \leq \frac{x}{2}$, and $r_{2}=\frac{p(q-2)}{3}+x-u$. For these replications, $x \leq p+\frac{u}{2}$ and

$$
\begin{aligned}
& \operatorname{int}\left[\frac{r_{1}}{q}\right]=\frac{p-1}{3}, \quad \operatorname{int}\left[\frac{r_{1}}{p}\right]=\left\{\begin{array}{l}
\frac{q+1}{3} \text { if } 0 \leq x \leq p \\
\frac{q-2}{3} \text { if } p<x \leq \frac{4 p-1}{3}
\end{array},\right. \\
& \operatorname{int}\left[\frac{r_{2}}{q}\right]=\operatorname{int}\left[\frac{r_{3}}{q}\right]=\frac{p-1}{3}, \quad \operatorname{int}\left[\frac{r_{2}}{p}\right]=\operatorname{int}\left[\frac{r_{3}}{p}\right]=\frac{q-2}{3}
\end{aligned}
$$

except at boundary points. There are two cases depending on the value of $\operatorname{int}\left[\frac{r_{1}}{p}\right]$. If $0 \leq x \leq p$, then using (3) and (4) gives

$$
\begin{gathered}
9 p q\left[(\sqrt{3}-1)\left(c_{2}+c_{3}\right)-c_{1}\right]=2\left(2 \sqrt{3} p q-2 \sqrt{3} p^{2} q+3 q^{2}-2 \sqrt{3} q^{2}-3 p^{2} q^{2}+2 \sqrt{3} p^{2} q^{2}-9 u^{2}\right. \\
\left.+9 \sqrt{3} u^{2}\right)+3(6 p-3 \sqrt{3} p-\sqrt{3} q+\sqrt{3} p q+6 u-6 \sqrt{3} u) x-9(2-\sqrt{3}) x^{2}
\end{gathered}
$$

which is a quadratic in $x$ with negative coefficient for $x^{2}$, so is positive if it is positive at the endpoints $x=0$ and $x=\frac{4 p-1}{3}$. For $x=0(\Rightarrow u=0)$ this expression is a quadratic in $q$, easily seen to be positive for all $q \geq 8$. For $x=\frac{4 p-1}{3}$ it is a quadratic in $u$ that attains its minimum at $u=\frac{4 p-1}{3}$; that value is $\frac{p-1}{3}(2 \sqrt{3}-3)\left[6 q^{2}(p+1)-3 q(2-\sqrt{3})-\sqrt{3}(4 p-1)\right]>0$. If $x>p$ then

$$
\begin{gathered}
9 p q\left[(\sqrt{3}-1)\left(c_{2}+c_{3}\right)-c_{1}\right]=2\left(-9 p^{2}+2 \sqrt{3} p q-2 \sqrt{3} p^{2} q+3 q^{2}-2 \sqrt{3} q^{2}-3 p^{2} q^{2}+2 \sqrt{3} p^{2} q^{2}\right. \\
\left.-9 u^{2}+9 \sqrt{3} u^{2}\right)+3(12 p-3 \sqrt{3} p-\sqrt{3} q+\sqrt{3} p q+6 u-6 \sqrt{3} u) x-9(2-\sqrt{3}) x^{2}
\end{gathered}
$$

It is easy to see that $(15)-(14)=18 p(x-p)>0$ since $x>p$. Thus (15) is also positive, and all nonuniform competitors are eliminated.

Step 3. The neighborhood determined in step 1 will now be further trimmed by eliminating all uniform designs with $\frac{p(q+1)}{3}<r_{1} \leq \frac{p(q+4)}{3}$. For a fixed $r_{3}=\frac{p(q-2)}{3}+u$ with $0 \leq u \leq \frac{p}{2}$ (since $\left.r_{3} \leq \frac{p q-r_{1}}{2}\right)$, write $r_{1}=\frac{p(q+1)}{3}+x$, and $r_{2}=p q-r_{1}-r_{3}=\frac{p(q+1)}{3}-x-u$, with $0 \leq x \leq p-2 u$ since $r_{2} \geq r_{3}$. With these $r_{i}$ the function $A$ defined in (10) is differentiable in $x$ everywhere. It will be 
shown that the derivative of $A$ with respect to $x$ is negative, thus forcing $r_{1} \leq \frac{p(q+1)}{3}$ for $A$-optimal designs.

$$
\begin{aligned}
\frac{\partial A}{\partial x} & =\left(c_{1}^{\prime}+c_{2}^{\prime}\right)-2 \frac{\left(2 c_{1} c_{1}^{\prime}+2 c_{2} c_{2}^{\prime}\right)\left(\sum c_{i}\right)-\left(c_{1}^{\prime}+c_{2}^{\prime}\right)\left(\sum c_{i}^{2}\right)}{\left(\sum c_{i}\right)^{2}} \\
& \stackrel{\operatorname{sign}}{=} \frac{27 p^{3} q^{3}}{8}\left\{\left(c_{1}^{\prime}+c_{2}^{\prime}\right)\left[\left(\sum c_{i}\right)^{2}+2 \sum c_{i}^{2}\right]-4\left(c_{1} c_{1}^{\prime}+c_{2} c_{2}^{\prime}\right)\left(\sum c_{i}\right)\right\}
\end{aligned}
$$

where $c_{i}^{\prime}=\frac{\partial c_{i}}{\partial x}\left(c_{3}\right.$ does not depend on $x$, so $\left.\frac{\partial c_{3}}{\partial x}=0\right)$. Evaluation of the $c_{i}$ and their derivatives requires the values of $\operatorname{int}\left[\frac{r_{i}}{p}\right]$ and $\operatorname{int}\left[\frac{r_{i}}{q}\right]$, which are

$$
\operatorname{int}\left[\frac{r_{1}}{p}\right]=\frac{q+1}{3}, \quad \operatorname{int}\left[\frac{r_{2}}{p}\right]=\operatorname{int}\left[\frac{r_{3}}{p}\right]=\frac{q-2}{3}, \quad \operatorname{int}\left[\frac{r_{1}}{q}\right]=\operatorname{int}\left[\frac{r_{2}}{q}\right]=\operatorname{int}\left[\frac{r_{3}}{q}\right]=\frac{p-1}{3},
$$

except at the boundary point $r_{1}=\frac{p(q+4)}{3}$. Using (3) and (4), (16) becomes a quadratic function $s_{2} x^{2}+s_{1} x+s_{0}$ in $x$ with coefficients

$$
\begin{aligned}
s_{2}= & 3 p q(p-1)(p q-q-3 u), \\
s_{1}= & -2 q^{2}(p-1)^{2}\left[-p^{2}+2 p q(p+1)-2 q^{2}(p+1)\right]+18 q(p-1)\left[p^{2}-p q(p+1)+q^{2}\left(p^{2}-1\right)\right] u \\
& -18 p[-3 p+4 q(p-1)] u^{2}+54(p q-p-q) u^{3}, \\
s_{0}= & -p q^{2}(p-1)^{2}(p q-p+q)^{2}+q(p-1)\left[8 p q^{2}-2 q^{3}+p^{3}\left(-9+13 q-8 q^{2}\right)+p^{2} q\left(2 q^{2}+5\right)\right] u \\
& +3\left[-9 p^{3}+12 p^{2} q(p-1)-2 p q^{2}(p-1)(2 p+1)+3 q^{3}(p+1)(p-1)^{2}\right] u^{2} \\
& -54 p(p q-p-q) u^{3}+27(p q-p-q) u^{4} .
\end{aligned}
$$

Coefficients $s_{2}$ and $s_{1}$ are positive for any $0 \leq u \leq \frac{p}{2}$ and $q \geq 2 p \geq 8$. Thus the derivative of this quadratic is an increasing function of $x$. Setting $x$ at its maximum value, which is $p-2 u$, produces a quartic polynomial in $u$ with coefficients $s_{j}$ of $u^{j}$ being

$$
\begin{aligned}
& s_{4}=-81(p q-p-q), \quad s_{3}=-\frac{4}{3} p s_{4}, \quad s_{2}=\frac{1}{9}\left(3 p^{2} q^{2}-3 q^{2}-p^{2} q+p q+3 p^{2}\right) s_{4}, \\
& s_{1}=3 q^{2}(p-1)\left(p^{2}-7 p^{3}-6 p q+6 p^{3} q+2 q^{2}-2 p^{2} q^{2}\right) \\
& s_{0}=-p q^{2}(p-1)^{2}\left(-4 p^{2}+2 p q+2 p^{2} q-3 q^{2}-2 p q^{2}+p^{2} q^{2}\right)
\end{aligned}
$$

The coefficients $s_{0}, s_{2}$, and $s_{4}$ are negative for any $q \geq 2 p \geq 8$. Also, $s_{2}+s_{3} u<0$ and $s_{0}+s_{1} u<0$ since $u \leq \frac{p}{2}$, so that the entire quartic is strictly negative. Thus the derivative of $A$ with respect to $x$ is negative, as claimed. 
Step 4. It remains to show that among uniform designs with replications $r_{i} \in\left[\frac{p(q-2)}{3}, \frac{p(q+1)}{3}\right], d^{*}$ is $A$-optimal. For a fixed $r_{1}=\frac{p(q+1)}{3}-u$ with $0 \leq u \leq \frac{p-1}{3}$, let $r_{3}=\frac{p(q-2)}{3}+x$ and so $r_{2}=\frac{p(q+1)}{3}+u-x$ with $2 u \leq x \leq \frac{p+u}{2}$, since $r_{1} \geq r_{2} \geq r_{3}$. It will be shown that $A$ is a decreasing function of $x$, thereby eliminating designs with $r_{1} \neq r_{2}$. Similar to (16), the derivative of $A$ with respect to $x$ is

$$
\frac{\partial A}{\partial x} \stackrel{\operatorname{sign}}{=} \frac{27 p^{3} q^{3}}{8}\left\{\left(c_{2}^{\prime}+c_{3}^{\prime}\right)\left[\left(\sum c_{i}\right)^{2}+2 \sum c_{i}^{2}\right]-4\left(c_{2} c_{2}^{\prime}+c_{3} c_{3}^{\prime}\right)\left(\sum c_{i}\right)\right\}
$$

For $\frac{p(q-2)}{3} \leq r_{i}<\frac{p(q+1)}{3}$, the integer-part functions needed in the $c_{i}$ are $\operatorname{int}\left[\frac{r_{i}}{p}\right]=\frac{q-2}{3}$ and $\operatorname{int}\left[\frac{r_{i}}{q}\right]=$ $\frac{p-1}{3}$. These with (3) and (4) show that (17) is a linear function of $x$, which can be written as $(p+u-2 x) S$, where $S$ is a cubic polynomial in $u$ with coefficients:

$$
\begin{aligned}
& s_{3}=27(p q+p-q), \quad s_{2}=-p s_{3}, \quad s_{1}=\frac{1}{3} q^{2}(p-1)(p+1) s_{3}, \\
& s_{0}=-q^{2}(p-1)^{2}\left(2 p q^{2}+2 q^{2}+p^{2} q+p q+p^{2}\right) .
\end{aligned}
$$

Simple algebra shows that $s_{0}+s_{1} u$ and $s_{2}+s_{3} u$ are negative for any $u \in\left[0, \frac{p-1}{3}\right]$. Thus $S$ is negative, and since $p+u-2 x \geq 0,(17)$ is negative for any $x$. This means that $A$ is a decreasing function of $x$, and so for a fixed $r_{1}$ the $A$-best designs have $x=2 u$ and consequently $r_{1}=r_{2}=\frac{p(q+1)}{3}-u$, $r_{3}=\frac{p(q-2)}{3}+2 u$ for some $u$.

It is here that the proof becomes counterintuitive. If the intuition from one-way blocking held, then maximizing $u$, that is, making the replications as equal as possible, would maximize $A$. To the contrary, Part I of this proof will be completed by showing that the best of the remaining competitors has $u=0$. That is, maximizing separation of the replications produces the best result.

So consider $A$ as a function of $u$ for uniform designs with the given replications. Depending on the values of $p$ and $q, A$ may or may not be monotonic in $u$. It will, however, be shown that the second derivative of $A$ with respect to $u$ is positive, so that $A$ is always convex in $u$. It will then be shown that $A$ is larger at $u=0$ than at $u=\frac{p-1}{3}$.

The derivative of $A$ with respect to $u$ is

$$
\frac{\partial A}{\partial u} \stackrel{\text { sign }}{=} \frac{9}{32} p^{3} q^{3}\left\{\left(2 c_{1}^{\prime}+c_{3}^{\prime}\right)\left[\left(\sum c_{i}\right)^{2}+2 \sum c_{i}^{2}\right]-4\left(2 c_{1} c_{1}^{\prime}+c_{3} c_{3}^{\prime}\right)\left(\sum c_{i}\right)\right\} \equiv g(u)
$$


where now $c_{i}^{\prime}=\frac{\partial c_{i}}{\partial u}$. Using (3) and (4) to expand the $c_{i}$ 's in (18) gives

$$
\begin{aligned}
g(u)= & -\frac{27}{2}(p q+p-q) u^{4}+18 p(p q+p-q) u^{3}-\frac{9}{2}\left(p^{2} q^{2}+p^{2}-q^{2}\right)(p q+p-q) u^{2}+q^{2}\left(p^{2}+2 p^{3}\right. \\
& \left.-2 p q+2 p^{3} q-q^{2}+p^{2} q^{2}\right)(p-1) u-\frac{1}{6} p q^{2}(p-1)^{2}\left(p^{2}+p q+p^{2} q+2 q^{2}+2 p q^{2}\right)
\end{aligned}
$$

Taking the derivative of $g$ with respect to $u$ gives a cubic polynomial in $u$ with coefficients $s_{j}$ of $u^{j}$ being

$$
\begin{aligned}
& s_{3}=-54(p q+p-q), \quad s_{2}=-p s_{3}, \quad s_{1}=\frac{1}{6}\left(p^{2} q^{2}+p^{2}-q^{2}\right) s_{3}, \\
& s_{0}=q^{2}(p-1)\left(p^{2} q^{2}-q^{2}+2 p^{3} q-2 p q+2 p^{3}+p^{2}\right) .
\end{aligned}
$$

Since $s_{0}+s_{1} u$ and $s_{2}+s_{3} u$ are each positive for any $0 \leq u \leq \frac{p-1}{3},(19)$ is increasing and $A$ is a convex function of $u$. Next, let $A^{*}$ and $A^{\prime}$ denote the function $A$ at $u=0$, and at $u=\frac{p-1}{3}$, respectively. Substituting in (10) using (3) and (4) and then simplifying yields $A^{*}-A^{\prime}=\frac{4(p-1)(p q-p+q)}{9 p q(p+1)(q-1)}>0$. This concludes part I of the proof.

Part II: $\mathbf{p}<\mathbf{q}<\mathbf{2 p}$. It follows from the $(\bmod 3)$ values of $p$ and $q$ that $p+1 \leq q \leq 2 p-3$. In this part some competitor designs will be eliminated by a uniform design, call it $d^{0}$, with replications $r_{1}^{0}=\frac{q(p+2)}{3}, r_{2}^{0}=r_{3}^{0}=\frac{q(p-1)}{3}$ and for which $c_{1}^{0}, c_{2}^{0}, c_{3}^{0}=c_{2}^{0}$ are the diagonal elements of the information matrix $C_{d^{0}} . C_{d^{0}}$ is identical to the corresponding row component information matrix since in $d^{0}$ columns are permutations of one another.

Let any competitor design $d$ have replications $r_{1} \geq r_{2} \geq r_{3}$. Step 1 of part I has already established that $r_{i} \in\left[\frac{p(q-2)}{3}, \frac{p(q+4)}{3}\right]$. The steps of the general strategy (13) are now implemented as

1. Eliminate all designs with $r_{1}>\frac{q(p+2)}{3}$, so that $r_{i} \in\left[\frac{p(q-2)}{3}, \frac{q(p+2)}{3}\right]$ for all $i$.

2. Eliminate all nonuniform designs with every $r_{i} \in\left[\frac{p(q-2)}{3}, \frac{q(p+2)}{3}\right]$.

3. Eliminate all remaining designs with $r_{3}<\frac{q(p-1)}{3}$.

4. Show that among uniform designs with replications $r_{i} \in\left[\frac{q(p-1)}{3}, \frac{q(p+2)}{3}\right], d^{*}$ is $A$-optimal.

Step 1. Consider a design $d$ with replication $r_{1}>\frac{q(p+2)}{3}$, and $c_{1}, c_{2}, c_{3}$ the diagonal elements of the row component design information matrix. Create the symmetrized design $\bar{d}$, which is at least as good as $d$, with $\bar{c}_{1}=c_{1}$ and $\bar{c}_{2}=\bar{c}_{3}=\frac{c_{2}+c_{3}}{2}$. Lemma 2.5 will show that $\bar{d}$ is $A$-inferior to $d^{0}$.

For condition $(i)$ of lemma 2.5, compute:

$$
2(\sqrt{3}-1) c_{2}^{0}-c_{1}^{0}=-\frac{2}{9}(\sqrt{3} q+\sqrt{3}-6)+\frac{2 p\left[(2 \sqrt{3}-3) q^{2}-2 \sqrt{3}\right]}{9 q}
$$


which is positive for any $q>p \geq 4$.

Condition (ii) of lemma 2.5 requires $\operatorname{trace}\left[C_{d^{0}}\right] \geq \operatorname{trace}\left[C_{\bar{d}}\right]=c_{1}+c_{2}+c_{3}$. By (11),

$c_{1}+c_{2}+c_{3}=p q-\frac{1}{q} \sum_{j=1}^{p}\left(n_{d 1 j}^{2}+n_{d 2 j}^{2}+n_{d 3 j}^{2}\right) \quad$ and $\quad c_{1}^{0}+2 c_{2}^{0}=p q-\frac{1}{q} \sum_{j=1}^{p}\left(n_{d^{0} 1 j}^{2}+n_{d^{0} 2 j}^{2}+n_{d^{0} 3 j}^{2}\right)$

where $n_{d i j}$ is the number of times treatment $i$ occurs in row $j$ for design $d$. To maximize $\operatorname{trace}\left[C_{\bar{d}}\right]$, take $d$ to be uniform in rows. Then $r_{1} \in\left[\frac{q(p+2)}{3}+1, \frac{p(q+4)}{3}\right]$ implies $n_{d 1 j} \in\left\{\frac{q+1}{3}, \frac{q+4}{3}\right\}$. Also, $r_{i} \geq \frac{p(q-2)}{3} \Rightarrow r_{3} \leq r_{2} \leq \frac{p(q+1)}{3} \Rightarrow n_{d i j} \in\left\{\frac{q-2}{3}, \frac{q+1}{3}\right\}$ for $i=2,3$. The $n_{d^{0} i j}$ fall in the same intervals. In a generalized binary design in rows, all of these counts are in $\left\{\frac{q-2}{3}, \frac{q+1}{3}\right\}$ and the sum of their squares has minimum possible value $p[h(q, 3)]$. For designs $d$ and $d^{0}$, the deviation from this minimum is two units for each unit that the largest replication is above $p(q+1) / 3$ :

$$
\sum_{j=1}^{p} \sum_{i=1}^{3} n_{d i j}^{2}=p[h(q, 3)]+2\left(r_{1}-\frac{p(q+1)}{3}\right), \quad \sum_{j=1}^{p} \sum_{i=1}^{3} n_{d^{0} i j}^{2}=p[h(q, 3)]+2\left(r_{1}^{0}-\frac{p(q+1)}{3}\right) .
$$

Since $r_{1}>r_{1}^{0}$, (ii) is established.

Lemma 2.5 (iii) requires $c_{2}^{0}>\bar{c}_{2}$. The quantity $2 \bar{c}_{2}=c_{2}+c_{3}$ is maximized if $d$ is uniform in rows, and then

$$
c_{2}+c_{3}=p q-r_{1}-\frac{1}{q} \sum_{i=1}^{2} \sum_{j=1}^{p} n_{d i j}^{2} \leq p q-r_{1}-\frac{2}{q} p\left(\frac{p q-r_{1}}{2 p}\right)^{2}=\frac{p q}{2}-\frac{r_{1}^{2}}{2 p q} .
$$

If $r_{1} \geq r_{1}^{0}+2$, then

$$
2 c_{2}^{0}-2 \bar{c}_{2} \geq \frac{2\left[(q+3)^{2}+2 p q-2 p^{2}\right]}{9 p q}>0
$$

If $r_{1}=r_{1}^{0}+1$, then

$$
2 c_{2}^{0}-2 \bar{c}_{2} \geq 2 c_{2}^{0}-\left(p q-r_{1}^{0}-1\right)+\frac{1}{q} h\left[p q-r_{1}^{0}-1,2 p\right]=\frac{q+1}{3 q}>0 .
$$

All conditions of lemma 2.5 are met, so all designs with $r_{1}>\frac{q(p+2)}{3}$ are eliminated.

Step 2. Next lemma 2.4 will be used to eliminate nonuniform designs with $r_{i} \in\left[\frac{p(q-2)}{3}, \frac{q(p+2)}{3}\right]$. So for any uniform design with replications in this neighborhood, it must be established that $(\sqrt{3}-1)\left(c_{2}+c_{3}\right)-c_{1}>0$. 
By lemma 2.1, $c_{i}$ is an increasing function of $r_{i}$ if $r_{i} \leq \frac{q(p-1)}{3}$. For $\frac{q(p-1)}{3} \leq r_{i} \leq \frac{q(p+2)}{3}, c_{i}$ is still an increasing function of $r_{i}$, since by (3),

$$
c_{i}\left(r_{i}+1\right)-c_{i}\left(r_{i}\right)=\left\{\begin{array}{lc}
\frac{1}{3 p q}\left(6 r_{i}-p q-q+p+3\right) & \text { if } \frac{q(p-1)}{3} \leq r_{i}<\frac{p(q+1)}{3} \\
\frac{1}{3 p q}\left(6 r_{i}-p q-q-5 p+3\right) & \text { if } \frac{p(q+1)}{3} \leq r_{i}<\frac{q(p+2)}{3}
\end{array} .\right.
$$

Thus, $c_{2} \geq c_{3} \geq c_{i}\left(\frac{p(q-2)}{3}\right)$ and $c_{1} \leq c_{i}\left(\frac{q(p+2)}{3}\right)$. Using (3) again,

$$
\begin{aligned}
2(\sqrt{3}-1) c_{i}\left(\frac{p(q-2)}{3}\right)-c_{i}\left(\frac{q(p+2)}{3}\right)= & -\frac{2}{9}(-15+10 \sqrt{3}-2 p+2 \sqrt{3} p)-\frac{4 p}{9 q} \\
& +\frac{2 q\left(-4+4 \sqrt{3}-p-3 p^{2}+2 \sqrt{3} p^{2}\right)}{9 p}
\end{aligned}
$$

which is an increasing function of $q$, positive if $q \geq p+4$ or if $p \geq 7$. The only $(p, q)$ pair that does not yield a positive number is $p=4$ and $q=5$. However, in this case all nonuniform designs can be eliminated numerically.

Step 3. Now the remaining designs with $r_{3}<\frac{q(p-1)}{3}$ will be eliminated. For a given $r_{1}=\frac{q(p+2)}{3}-u$ with $0 \leq u \leq \frac{q}{2}$, write $r_{3}=\frac{q(p-1)}{3}-x$ and $r_{2}=p q-r_{1}-r_{3}=\frac{q(p-1)}{3}+x+u$ with $0 \leq x \leq$ $\min \left(q-2 u, \frac{2 p-q}{3}\right)$, where the restrictions for $u$ and $x$ follow directly from $r_{1} \geq r_{2} \geq r_{3} \geq \frac{p(q-2)}{3}$. Similar to step 3 of Part I, the derivative of the function $A$ defined in (10) with respect to $x$ can be shown to be negative. This is equivalent to establishing negativity of the expression (17) for the $c_{i}$ 's here. Depending on the value of $\operatorname{int}\left[\frac{r_{1}}{p}\right]$, there are two forms that (17) can take, both quadratic in $x$ with coefficients $s_{j}$ of $x^{j}$ having $s_{2}>0$. With arguments similar to those in step 3 of Part I, each of these quadratics can be shown to be negative for every $u$ at the endpoints for $x$ (details omitted in deference to space). Thus (17) is negative for any $x$ and $u$. Henceforth, only uniform designs with $r_{i} \in\left[\frac{q(p-1)}{3}, \frac{q(p+2)}{3}\right]$ need to be considered.

Step 4. For the remaining competitors, it will first be shown that for a given $r_{1}$ the $A$-best design has minimum $r_{3}$. Then it will be shown that among such designs, the $A$-best design is $d^{*}$.

Similar to the previous step, write $r_{1}=\frac{q(p+2)}{3}-u$ with $0 \leq u \leq \frac{2 q-1}{3}, r_{3}=\frac{q(p-1)}{3}+x$, and $r_{2}=p q-r_{1}-r_{3}=\frac{q(p-1)}{3}-x+u$ with $0 \leq x \leq \frac{u}{2}$. Also, $x \geq 2 u-q$ for any given $u$; restrictions for $u$ and $x$ follow directly from $r_{1} \geq r_{2} \geq r_{3}$ and $r_{i} \in\left[\frac{q(p-1)}{3}, \frac{q(p+2)}{3}\right]$. It will be shown that $A$ is a decreasing function of $x$. Like in step 3, this is equivalent to establishing negativity of the expression (17) for the $c_{i}$ 's here. Again, (17) has two different forms depending on int $\left[\frac{r_{1}}{p}\right]$. Both are 
linear in $x$ with positive coefficient for $x$, and both are zero at $x=\frac{u}{2}$ (details again omitted). Thus, for given $r_{1}, A$-best designs have minimum $r_{3}$. This is $r_{3}=\frac{q(p-1)}{3}$ if $r_{1} \geq \frac{p q-q(p-1) / 3}{2}=\frac{q(2 p+1)}{6}$, and $r_{3}=p q-2 r_{1}$ if $r_{1}<\frac{q(2 p+1)}{6}$.

To find the $A$-optimal design among the remaining competitors, write $r_{1}=\frac{q(p+2)}{3}-x$. It will be shown that $A$ is increasing in $x$ for $x \leq \frac{q}{2}$, and decreasing for $x \geq \frac{q}{2}$.

First, for $x \leq \frac{q}{2}$ the other replication numbers are $r_{2}=\frac{q(p-1)}{3}+x$ and $r_{3}=\frac{q(p-1)}{3}$. The sign of the derivative of $A$ with respect to $x$ is now the sign of (16) evaluated for the $c_{i}$ 's here. Again, this expression takes two forms depending on $\operatorname{int}\left[\frac{r_{1}}{p}\right]$.

If $x \leq \frac{2 q-p}{3}$, then (16) is a quadratic function of $x$ with coefficients

$$
\begin{aligned}
& s_{2}=-3 p^{2} q(p-1)(q-2), \\
& s_{1}=2 p^{2}\left[4 p^{2}+\left(7-4 p-6 p^{2}\right) q^{2}+\left(-4+p+4 p^{2}\right) q^{3}+(1-p) q^{4}\right], \\
& s_{0}=p^{2}\left[4 p^{3}-4 p^{2} q+\left(3 p-6 p^{3}\right) q^{2}+\left(-7+2 p+7 p^{2}+2 p^{3}\right) q^{3}+\left(4-6 p^{2}+p^{3}\right) q^{4}+(p-1) q^{5}\right] .
\end{aligned}
$$

The coefficient $s_{2}$ of $x^{2}$ is negative, and this quadratic is positive both at $x=0$ and $x=\frac{q}{2}$, so (16) is positive. If $x>\frac{2 q-p}{3}$, then (16) reduces to a linear function of $x$ with coefficients

$$
s_{1}=-2 p^{2}(q+1)^{2}\left(p q^{2}-q^{2}+2 p^{2} q-p q-2 p^{2}\right), \quad s_{0}=-\frac{q}{2} s_{1}
$$

This is decreasing in $x$ and is 0 at $x=\frac{q}{2}$, so (16) is positive, and $A$ is increasing in $x$, for any $x \leq \frac{q}{2}$. Now suppose $x>\frac{q}{2}$, so that $r_{2}=r_{1}=\frac{q(p+2)}{3}-x, r_{3}=p q-2 r_{1}=\frac{q(p-4)}{3}+2 x$, and also $c_{1}=c_{2}$. For these values $\frac{\partial A}{\partial x}$ can be expressed as

$$
\frac{\partial A}{\partial x} \stackrel{\operatorname{sign}}{=} \frac{27 p^{3} q^{3}}{16}\left[c_{3}^{\prime}\left(8 c_{1}^{2}-4 c_{1} c_{3}-c_{3}^{2}\right)+6 c_{3}^{2} c_{1}^{\prime}\right]
$$

which when simplified using (3) is the quartic function $\sum_{j=0}^{4} s_{j} x^{j}$ with

$$
\begin{aligned}
s_{4}= & -81(p q+p-q), \quad s_{3}=-\frac{8}{3} q s_{4}, \quad s_{2}=\frac{1}{3}\left(p^{2} q^{2}+7 q^{2}-p^{2}\right) s_{4}, \\
s_{1}= & -6\left[-p^{4}+\left(7 p^{3}-p^{4}\right) q+\left(-8 p^{2}+7 p^{3}+p^{4}\right) q^{2}+\left(-9 p-p^{2}-7 p^{3}+p^{4}\right) q^{3}\right. \\
& \left.+\left(9-9 p+7 p^{2}-7 p^{3}\right) q^{4}\right], \\
s_{0}= & -4 p^{2} q(q+1)\left(4 p q^{3}-4 q^{3}-p^{2} q^{2}+5 q^{2}-4 p q+p^{2}\right) .
\end{aligned}
$$


Coefficient $s_{4}$ is negative, and $s_{2}+3 s_{3} x<0$ for any $x \in\left[\frac{q}{2}, \frac{2 q-1}{3}\right]$ and $q \geq p+1 \geq 5$. Hence, this quartic polynomial must be concave; also its first derivative is positive at $x=\frac{2 q-1}{3}$, and so the quartic is increasing in $x$. But it is negative at $x=\frac{2 q-1}{3}$, and so (20) is negative for any allowable $x$, that is, $A$ is decreasing in $x>\frac{q}{2}$.

In summary, the optimizing value for $x$ is $x=\frac{q}{2}$, and it is obvious that when $q$ is even, $d^{*}$ with $r_{1}^{*}=r_{2}^{*}=\frac{q(2 p+1)}{6}, r_{3}^{*}=\frac{q(p-1)}{6}$ is $A$-optimal. But if $q$ is odd, $\frac{q(2 p+1)}{6}$ is not an integer. Two competitors remain:

$$
\begin{array}{lll}
d^{\prime} \quad \text { with } & r_{1}=r_{2}=\frac{q(2 p+1)-3}{6}, \quad r_{3}=\frac{q(p-1)}{3}+1, \text { and } \\
d^{*} \quad \text { with } \quad r_{1}=\frac{q(2 p+1)+3}{6}, \quad r_{2}=\frac{q(2 p+1)-3}{6}, \quad r_{3}=\frac{q(p-1)}{3} .
\end{array}
$$

To show that $d^{*}$ is $A$-superior to $d^{\prime}$, simply use (10) and (3) to compute $A_{d^{*}}-A_{d^{\prime}}>0$.

\section{Discussion}

Many practicing scientists gain their design knowledge from statistics texts, which typically limit coverage of row-column settings to Latin squares and Youden designs. Consequently these "standard" designs are sometimes employed by modifying one or more of $v, p$, and $q$ relative to original intent and total available resources. But experimental practice should be limited only by the resources, not by inadequate lists of recommended options. This cannot change until statistical theory gets a better handle on the row-column problem, so that fuller, more flexible design catalogs can be made available. This work is one step in that direction. Construction of designs meeting the conditions of Theorem 4.1 is solved in section 4.4.2 of Parvu (2004).

While three treatments is of limited scope relative to the $(v, p, q)$ universe, it does have important practical implications. As one example, row-column designs are sometimes employed for repeated measures experiments (row=subject, column=time period), requiring only that adequate washout intervals are provided to negate carry-over effects. The designs here can be used for comparing three drugs, say, for whatever numbers of subjects and time periods that a researcher can muster. Rowcolumn designs appear in many other applications, including agricultural field experimentation, greenhouse experiments, and growth chamber trials in which rows and columns correspond to 
spatial distribution of experimental units. The results here offer full flexibility in design whenever three treatments are to be compared, be they genetically modified varieties, competing management practices, pesticide rates, fertilizing dates, or any of a plethora of other possibilities.

It is worth mentioning that the lemmas of section 2 are applicable not just for row-column designs, but for any $3 \times 3$ information matrix with zero row and column sums. They can thus be used, for example, in the study of optimal 3-treatment designs with more than two blocking factors, and/or with correlated errors.

Parvu and Morgan (2005) investigated the same problem as here from an E-optimlity perspective. In contrast to Theorem 4.1, they found that E-optimal designs must have replications as equal as possible, but in many cases are nonuniform. Both results stand in contrast to Theorem 3.1, in that the row and column component designs need not be the optimal block designs identified there. Taken all together, these results make abundantly clear the technical intricacy of row-column design optimality. It is conjectured that, depending on $p$ and $q$, similar behaviors will be found for any $v$.

\section{ACKNOWLEDGEMENT}

Our thanks to the referees for comments enhancing the presentation. J. P. Morgan was supported by National Science Foundation grant DMS01-04195.

\section{REFERENCES}

BAGCHI, S. (1996). An infinite series of adjusted orthogonal designs with replication two. Statistica Sinica 6, 975-987.

Bagchi, S. And Shah, K. R. (1989). On the optimality of a class of row-column designs. Journal of Statistical Planning and Inference 23, 397-402.

Bagchi, S. And van Berkum, E. E. M. (1991). On the optimality of a new class of adjusted orthogonal designs. Journal of Statistical Planning and Inference 28, 61-65.

Cheng, C.-S. (1979a). Optimal incomplete block designs with four varieties. Sankhyā B 41, 1-14.

Cheng, C.-S. (1979b). Construction of Youden Hyperrectangles. Journal of Statistical Planning and Inference 3, 109-118. 
Cheng, C.-S. (1981). Optimality and construction of pseudo-Youden designs. Annals of Statistics 9, 201-205.

DAs, A. (1993). E-optimal block and row-column designs with unequal number of replicates. Sankhyā B 55, 77-90.

DAs, A. AND Dey, A. (1992). Universal optimality and nonoptimality of some row-column designs. Journal of Statistical Planning and Inference 31, 263-271.

Constantine, G.M. (1981). Some E-optimal Block Designs. Annals of Statistics 9, 886-892.

Jacroux, M. (1985). Some E and MV-optimal designs for the two-way elimination of heterogeneity. Annals of the Institute of Statistical Mathematics 37, 557-566.

Jacroux, M. (1986). Some E-optimal row-column designs. Sankhyā B 48, 31-39.

JACROUX, M. (1987). Some E and MV-optimal row-column designs having equal numbers of rows and columns. Metrika 6, 361-381.

JACROUX, M. (1990). Some E-optimal row-column designs having unequally replicated treatments. Journal of Statistical Planning and Inference 26, 65-81.

Kiefer, J. (1975). Construction and optimality of generalized Youden designs. In A Survey of Statistical Design and Linear Models, Ed. J.N. Srivastava, pp. 333-353. North-Holland, Amsterdam.

Magda, C. G. (1980). Circular balanced repeated measurements designs. Comm. Statist. Theory Methods 9, 1901-1918.

Marshall, A.W. And Olkin, I. (1979). Inequalities: Theory of Majorization and Its Applications. Academic Press, New York.

Morgan, J. P. (1997). On pairs of Youden designs. Journal of Statistical Planning and Inference 60, 367-387.

Morgan, J.P. And Bailey, R .A. (2000). Optimal design with many blocking factors. Annals of Statistics 28, 553-577. 
PARVU, V. (2004). Optimal blocking for three treatments and BIBD robustness: two problems in design optimality. Ph.D. Dissertation, Virginia Polytechnic Institute and State University, Blacksburg. http://scholar.lib.vt.edu/theses/available/etd-12032004-163822/unrestricted/dissertation.pdf. Parvu, V. And Morgan, J.P. (2005). E-optimal Designs for Three Treatments. Submitted.

Shah, K. R. And Sinha, B. K. (1989). Theory of Optimal Designs. Springer-Verlag, New York. Singh, R. And Gupta, V. K. (1991). E-optimal designs for two-way elimination of heterogeneity. Sankhyā B 53, 97-104.

Sonnemann, E. (1985). U-optimum row-columns designs for the comparison of two treatments. Metrika 32, 57-63. 